\title{
Biallelic mutations in CYP24A1 or SLC34A1 as a cause of infantile idiopathic hypercalcemia (IIH) with vitamin D hypersensitivity: molecular study of 11 historical IIH cases
}

\author{
Ewa Pronicka $^{1,2} \cdot$ Elżbieta Ciara $^{2} \cdot$ Paulina Halat $^{2}$ - Agnieszka Janiec ${ }^{1}$. \\ Marek Wójcik $^{3}$ - Elżbieta Rowińska ${ }^{1}$ - Dariusz Rokicki ${ }^{1}$. Pawel Płudowski ${ }^{3}$. \\ Ewa Wojciechowska ${ }^{4}$ • Aldona Wierzbicka ${ }^{3}$. Janusz B. Książyk ${ }^{1}$. \\ Agnieszka Jacoszek $^{5,6} \cdot$ Martin Konrad $^{7} \cdot$ Karl P. Schlingmann $^{7} \cdot$ Mieczyslaw Litwin $^{4}$
}

Received: 22 December 2016/Revised: 19 March 2017 / Accepted: 17 April 2017 / Published online: 3 May 2017

(C) The Author(s) 2017. This article is an open access publication

\begin{abstract}
Idiopathic infantile hypercalcemia (IIH) is a mineral metabolism disorder characterized by severe hypercalcemia, failure to thrive, vomiting, dehydration, and nephrocalcinosis. The periodical increase in incidence of IIH, which occurred in the twentieth century in the United Kingdom, Poland, and West Germany, turned out to be a side effect of rickets over-prophylaxis. It was recently discovered that the condition is linked to two genes, CYP24A1 and SLC34A1. The aim of the study was to search for pathogenic variants of the genes in adult persons who were shortlisted in infancy as IIH caused by "hypersensitivity to vit. D". All persons were found to carry mutations in CYP24A1 or SLC34A1, nine and two persons respectively. The changes
\end{abstract}

All authors contributed equally to this work.

Communicated by: Michal Witt

Ewa Pronicka

e.pronicka@ipczd.pl

1 Department of Pediatrics, Nutrition and Metabolic Diseases, The Children's Memorial Health Institute, Aleja Dzieci Polskich 20, 04-730 Warsaw, Poland

2 Department of Medical Genetics, The Children's Memorial Health Institute, Warsaw, Poland

3 Department of Biochemistry, Radioimmunology and Experimental Medicine, The Children's Memorial Health Institute, Warsaw, Poland

4 Department of Nephrology, The Children's Memorial Health Institute, Warsaw, Poland

5 Department of Medical Genetics, Warsaw Medical University, Warsaw, Poland

6 Postgraduate School of Molecular Medicine, Warsaw, Poland

7 University Children's Hospital, Münster, Germany were biallelic, with one exception. Incidence of IIH in Polish population estimated on the basis of allele frequency of recurrent p.R396W CYP24Al variant, is 1:32,465 births. It indicates that at least a thousand homozygotes and compound heterozygotes with risk of IIH live in the country. Differences in mechanism of developing hypercalcemia indicate that its prevention may vary in both IIH defects. Theoretically, vit. D restriction is a first indication for CYP24A1 defect (which disturbs $1,25(\mathrm{OH})_{2} \mathrm{D}$ degradation) and phosphate supplementation for SLC34A1 defect (which impairs renal phosphate transport). In conclusion, we suggest that molecular testing for CYP24A1 and SLC34A1 mutations should be performed in each case of idiopathic hypercalcemia/hypercalciuria, both in children and adults, to determine the proper way for acute treatment and complications prevention.

Keywords Idiopathic infantile hypercalcemia $\cdot$ Vitamin D hypersensitivity - Biallelic mutations - CYP24A1 S SLC34A1 . Adults

\section{Introduction}

Idiopathic infantile hypercalcemia (IIH) first received attention in the 1950s in the UK because of a sudden increase in the incidence. A number of years passed until it turned out to be a side effect of a just started program of rickets prophylaxis. Reducing vitamin D (vit. D) food supplementation normalized the situation (Samuel 1964).

We experienced a similar "endemic" condition in the 1970s in Poland (Pronicka et al. 1985), in the period of commonly administered so called "periodic" vit. D dosage (300,000 units, 3 times per year). The same phenomenon was observed in Eastern Germany (Misselwitz et al. 1990). At least 36 infants 
with IIH from the entire country were treated in our pediatric reference centre (Pronicka et al. 1985, 1988, 1997; Rowińska et al. 1998). During the normocalcemic phase of the disease, on continuous vit. D restriction, we found relatively high 25 hydroxyvitamin D (25OHD) and 1,25-dihydroxyvitamin D $\left(1,25(\mathrm{OH})_{2} \mathrm{D}\right)$ concentrations. The condition was named "hypersensitivity to vitamin D", to distinguish it from Williams syndrome and from other, non-vitamin D sensitive IIH types (Pronicka et al. 1988, 1997). Autosomal recessive inheritance was anticipated, with frequency about 1:60,000 births.

Genetic inheritance of the IIH was confirmed only recently by the discovery of two responsible genes CYP24A1 and SLC34A1 (Schlingmann et al. 2011, 2016). Biallelic mutations in these genes have a completely different mechanism leading to (causing) hypercalcemia (Fig. 1). In the first CYP24A1 defect, hypercalcemia is directly caused by impaired degradation of $1,25(\mathrm{OH})_{2} \mathrm{D}$ (Schlingmann et al. 2011). In the SLC34Al defect, hypercalcemia is a sequel of primary loss of phosphate in the kidney followed by downregulation of FGF-23, which in-turn leads to increased vit. D activity (Schlingmann et al. 2016).

The aim of the study was to search for pathogenic variants of CYP24A1 and SLC34A1 in adult persons who were shortlisted in infancy as "hypersensitive to vit. D". The majority of them were described in childhood (Pronicka et al. 1985, 1997; Misselwitz et al. 1990).

\section{Patients and methods}

There were two criteria for the study recruitment: (1) historical diagnosis of "vit. D hypersensitity" and (2) current adult age of the invited patients. Informed consent for DNA analysis was given by 11 patients out of $17(64.7 \%)$ to whom a registered letter of invitation was successfully delivered.

All 11 patients included in the study were qualified as "vit. D hypersensitive" depending on the common features used by us in the 1970-1990s, as follows: (1) temporal link between appearance of hypercalcemia and administration of vit. D; (2) low PTH level (exclusion of hyperparathyroidism); (3) increased echogenicity of renal pyramids on ultrasound; (4) sustained tendency to hypercalcemia; (5) immediate response to steroid treatment, and/or low calcium diet. They presented in infancy with loss of appetite, failure to thrive, arrest in psychomotor development, constipation, and dehydration. Some patients needed several months of hospital treatment. Over childhood, the patients remained under the care of the calcium-phosphate clinic (ER) in our institute for 2-18 years (median value 14 years). Gradual normalization of clinical status and catch up in growth were observed after recovery from the episode of hypercalcemic decompensation in infancy. During the development period some of the children needed orthopedic intervention and rehabilitation due to mild dysplastic changes of hips, knees or feet. Management, at that
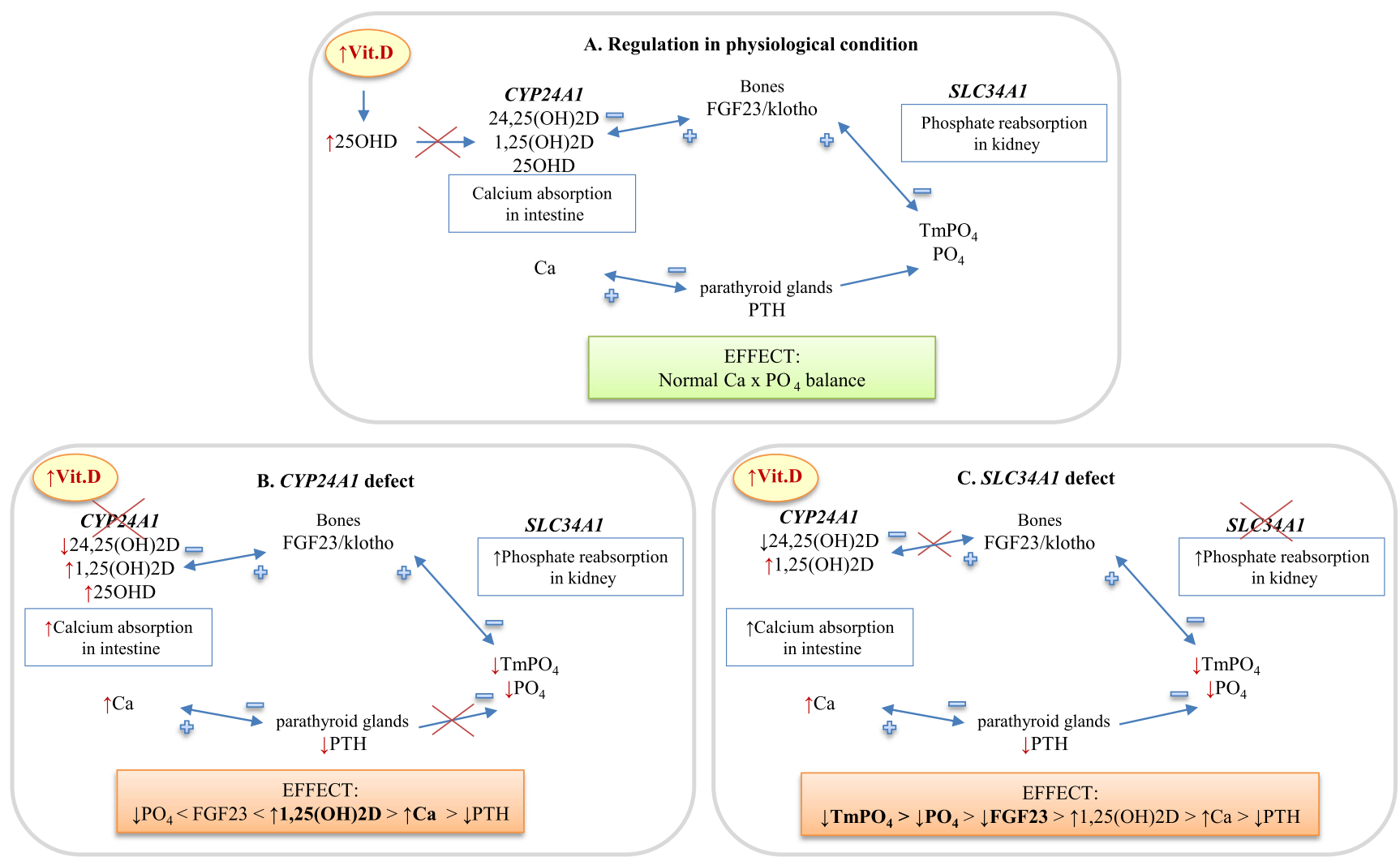

Fig. 1 Simplified scheme of hypothetical regulation of calcium-phosphate balance in vitamin D overload status 
time, included: permanent withdrawal of vit. D supply and sun protection, dietary calcium restriction, and increased fluid intake. Despite the above prophylaxis, the 25-OHD concentration maintained within high/normal limits, and laboratory data showed a tendency to hypercalcemia, hypercalciuria, and relatively low phosphate levels throughout childhood (Table 1).

In nine patients Sanger sequencing analysis was carried out in Muenster according to the protocol described previously (Schlingmann et al. 2011, 2016). In two patients (P7 and P9) next-generation sequencing (NGS) was conducted. NGS was performed on a HiSeq 1500 using TruSight One Sequencing Panel (Illumina) according to the manufacturer's instructions. Generated reads were aligned to the hg19 reference human genome. The detected variants were annotated using Annovar (Wang et al. 2010) and converted to MS Access format for final manual analyses. Bioinformatics analysis was performed as previously described (Ciara et al. 2016). Alignments were viewed with Integrative Genomics Viewer v.2.2.79 (Robinson et al. 2011). Mutation numbering was based on the cDNA sequence (human CYP24A1, GenBank NM_000782.4; human SLC34A1, GenBank NM_003052.4) according to the guidelines of Human Genome Variation Society (HGVS, www.hgvs.org/mutnomen).

Molecular analysis was performed after obtaining written informed consent.

\section{Results and discussion}

At the study beginning, all patients were working or studying. They were in very good health and without complaints. Mean weight, height, and body mass index (BMI) were respectively $180.7 \pm 10.1 \mathrm{~cm}, 83.4 \pm 31.6 \mathrm{~kg}$, and $25 \pm 7$ for males and $164.7 \pm 2.6 \mathrm{~cm}, 63.7 \pm 9.1 \mathrm{~kg}$, and $23.5 \pm 3.8$ for females. Three patients were under the control of nephrologist in the place of residence, due to sustained nephrocalcinosis (stable from infancy). Urolithiasis and progressive renal failure, reported in IIH (Molin et al. 2015) did not occur in any of the patients included in molecular study. Serum creatinine concentration measured in two patients was at bordeline of the control values $(1.29,1.13 \mathrm{mg} / \mathrm{dl})$.

The results of molecular studies are shown in Table 1. In all 11 adults, the historical diagnosis of IIH was confirmed at the molecular level with $100 \%$ compliance. We detected pathogenic variants in one of two genes studied, CYP24A1 in nine patients and $S L C 34 A 1$ in two remaining ones. The latter subset was already included in the original publication (Schlingmann et al. 2016).

In CYP24A1, two novel variants c.107del (p.P36Lfs*11) and c. $1157+1 \mathrm{G}>\mathrm{A}(\mathrm{p}$. ?) as well as five known variants [c.428_430del (p.E143del), 443 T > C (p.L148P), c.964G > A (p.E322K), c.1186C > T (p.R396W), c.1226 T > C (p.L409S)] were identified in homozygous or compound heterozygous state
(Table 1). Missense variant p.R396W occurred in seven of nine CYP24A1 mutated persons (11/18 alleles; four homozygotes and three compound heterozygotes). Previously this variant, found in four of 1024 control alleles, was annotated as putative polymorphism; however, it finally concluded as pathogenic (Schlingmann et al. 2011).

Four p.R396W alleles were found in 588 samples (1176 alleles) from general Polish population. Based on this data the frequency of p.R396W allele was calculated as 0.0034 (0.34\%; 1:294). Using the Hardy-Weinberg's law the frequency of p.R396W carriers in the Polish population was estimated as $0.0068(0.68 \% ; 1: 147)$. Taking into account that p.R396W make up approximately $61.1 \%$ of CYP24A1 alleles, overall carrier frequency for all mutation in CYP24Al was calculated as $1.11 \%$ (1:90). Thus, the expected incidence of CYP24A1 deficient IIH in Poland was estimated as 1:32,465 births (with 0.95 confidence interval). A thousand potentially affected CYP24A1 deficient persons may be living in the country.

The study group of IIH, at start considered genetically homogeneous, turned out to be divided in two. Laboratory data was re-analyzed in two subsets of patients, with CYP24A1 and of SLC 34A1 defects. Age of onset, total/daily vitamin D dose, duration of hypercalcemia, prednisone treatment, milk free diet, calcium/phosphate concentrations and excretion, $25 \mathrm{OHD}, 1,25(\mathrm{OH})_{2} \mathrm{D}$, and PTH levels were assessed separately (Table 1). We were not able to identify any unequivocal feature useful to distinguish CYP24A1 from SLC34A1 defect at presentation of hypercalcemia in infancy. Only two potentially important differences have to be mentioned: (1) the previously reported finding that $25 \mathrm{OHD}$ concentration in IIH probands, carriers of two mutated variants, was twice as high as in their parents, carriers of a single mutated allele (Rowińska et al. 1998), was observed only in the families with CYP24A1 defect, (2) kidney stones were found in one father of an IIH proband (Rowińska et al. 1998) with SLC34AI defect. Unfortunately those old findings are anecdotal (data no longer available) and have to be verified in the future study.

Despite the fact that, in the light of present knowledge, our historical diagnosis of "hypersensitivity to vitamin D" is correct for both, the CYP24A1 and the SLC34A1 defects, the identical treatment options may require a revision. We may speculate, e.g., that the long-term calcium restriction and vit. $\mathrm{D}$ avoidance might be incorrect and possibly harmful for the patients with SLC34A1 defect (P10 and P11). Alternative treatment with phosphates supplementation should be considered and evaluated. By the way, increased phosphate intake was recommended constantly for our patients with $\mathrm{IIH}$, and was even tested in a short experiment (Rowińska et al. 1998), however no differences in response to phosphates between individual patients were observed and no conclusions were then attained.

This lack of laboratory difference is likely due to strict control of the calcium-phosphate homeostasis to avoid the negative effects of vitamin $\mathrm{D}$ excess on concentration of both 


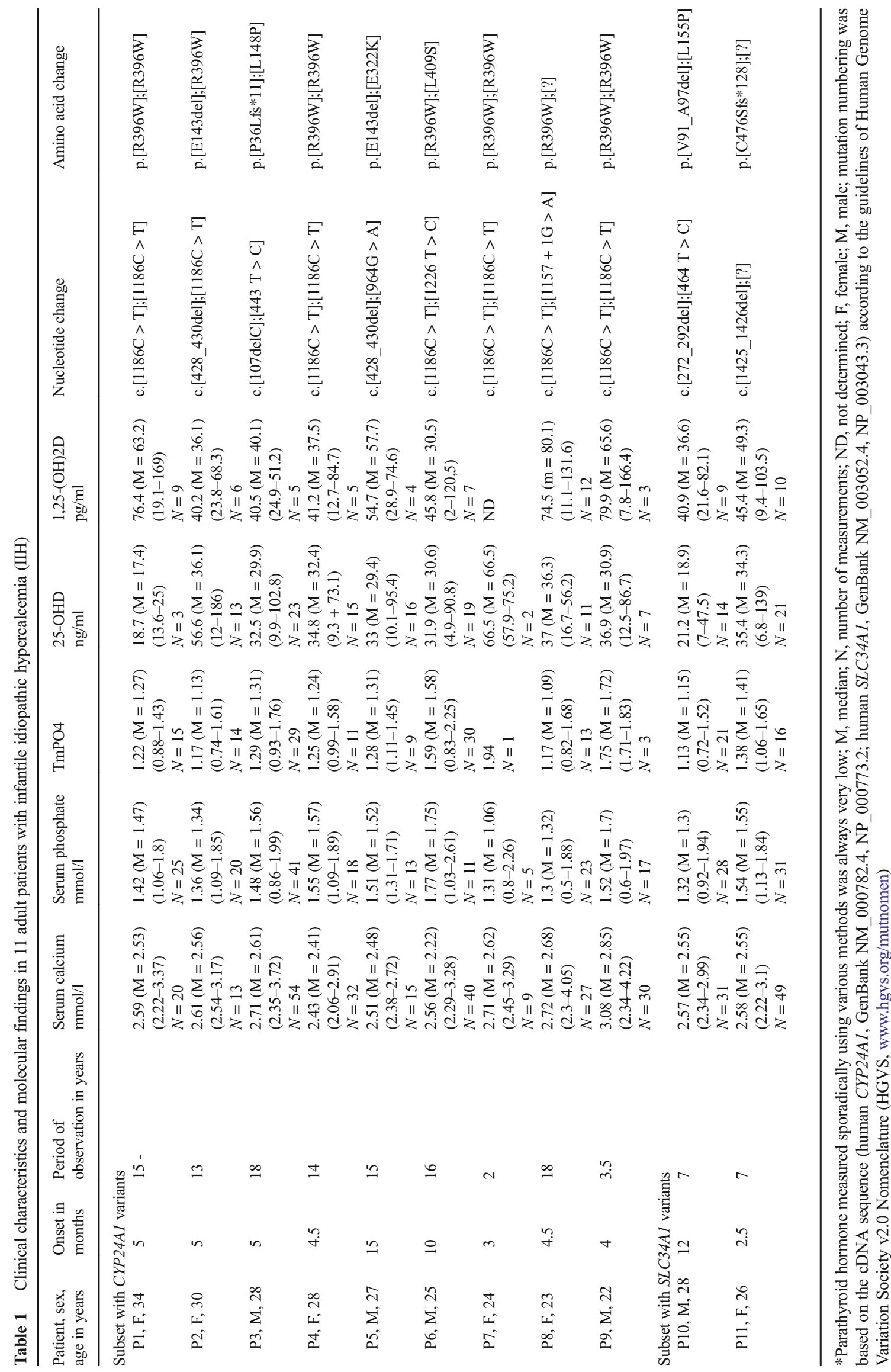


calcium and phosphate during mineralization. In such a situation the optimal condition for mineralization requires feedback reaction in the direction opposite to primary genetic defect (e.g., calcium absorption in SLC34A1 and phosphate reabsorption in CYP24A1). As a result, the final effect of elevated $1,25(\mathrm{OH})_{2} \mathrm{D}$ is similar in both SLC34Al and CYP24Al defects (Fig. 1). It seems obvious, however, that counteracting the consequences of both defects may be different; hence, the need to establish the molecular background of IIH is crucial, not only in infants but also in adults. Our study indicates that the IIH defect is relatively frequent, and if not detected, is potentially dangerous also for adults.

The discovery of the association between IIH and the mutations in SLC34A1 and CYP24A1 genes opened a new research area. Several questions are under investigation, among them:

1. Are CYP24A1 and SLC34A1 defects neutral for health if vit. $D$ is not excessively supplemented?

2. Whether and how CYP24A1 and SLC34A1 mutations contribute to modifying bone growth and calcification; are they involved in nephrolithiasis development?

3. Whether benefits of long-term calcium/vit. D restriction in IIH infants outweigh adverse events in case of CYP24A1 and SLC 34A1 mutations?

4. What is the actual prevalence of CYP24A1 and SLC34A1 mutations and what is the risk throughout life of being a carrier of biallelic mutations in these genes?

5. How often do these defects in the population occur, and if indeed there are any ethnic differences?

6. Whether and on what basis can CYP24A1 and SLC34AI mutations in these defects be distinguished without molecular testing (e.g, measure of 24OHD)?

Acknowledgements The authors express their gratitude to all patients and their families for the willingness to participate in the study. We appreciate the invaluable contribution of nurses and we would like to thank the laboratory and administrative staff of CMHI for their help in complying with the study protocol.

We would like to heartily thank other collaborators of the Children's Memorial Health Institute, Warsaw, especially Mrs. Dorota Siestrzykowska from the Department of the Medical Genetics for excellent technical assistance.

Compliance with ethical standards This study was supported by projects: NSC 2014/15/B/NZ5/03541, CMHI 180/09.

\section{Conflict of interest None.}

Ethical approval All procedures performed in studies involving human participants were in accordance with the ethical standards of the Bioethical Committee at the Children's Memorial Health Institute of Warsaw and with the 1964 Helsinki declaration and its later amendments or comparable ethical standards.

Informed consent Informed consent was obtained from all individual participants included in the study.
Open Access This article is distributed under the terms of the Creative Commons Attribution 4.0 International License (http:// creativecommons.org/licenses/by/4.0/), which permits unrestricted use, distribution, and reproduction in any medium, provided you give appropriate credit to the original author(s) and the source, provide a link to the Creative Commons license, and indicate if changes were made.

\section{References}

Ciara E, Rokicki D, Halat P, Karkucinska-Wieckowska A, PiekutowskaAbramczuk D, Mayr J, Trubicka J, Szymanska-Debinska T, Pronicki M, Pajdowska M, Dudzinska M, Gizewska M, Krajewska-Walasek M, Ksiazyk J, Sperl W, Ploski R, Pronicka E (2016) Difficulties in recognition of pyruvate dehydrogenase complex deficiency on the basis of clinical and biochemical features. The role of next-generation sequencing. Mol Genet Metab Rep 7:70-76

Misselwitz J, Hesse V, Markestad T (1990) Nephrocalcinosis, hypercalciuria and elevated serum levels of 1,25-dihydroxyvitamin D in children. Possible link to vitamin D toxicity. Acta Paediatr Scand 796-7:637-643

Molin A, Baudoin R, Kaufmann M, Souberbielle JC, Ryckewaert A, Vantyghem MC, Eckart P, Bacchetta J, Deschenes G, KeslerRoussey G, Coudray N, Richard N, Wraich M, Bonafiglia Q, Tiulpakov A, Jones G, Kottler ML (2015) Mutations in a cohort of hypercalcemic patients: evidence for a recessive trait. J Clin Endocrinol Metab 100(10):E1343-E1352

Pronicka E, Kulczycka H, Rowinska E, Konopinska A, Kansy J, Lorenc $\mathrm{R}$ (1985) Idiopathic hypercalcemia as a syndrome of hypersensitivity to vitamin D3 in 19 infants. Pediatr Pol 604:288-294

Pronicka E, Kulczycka H, Lorenc R, Proszynska K, Gradzka J, Rowinska E (1988) Increased serum level of 1,25-dihydroxyvitamin D3 after parathyroid hormone in the normocalcemic phase of idiopathic hypercalcemia. J Pediatr 1126:930-933

Pronicka E, Rowinska E, Kulczycka H, Lukaszkiewicz J, Lorenc R, Janas R (1997) Persistent hypercalciuria and elevated 25-hydroxyvitamin D3 in children with infantile hypercalcaemia. Pediatr Nephrol 111:2-6

Robinson JT, Thorvaldsdottir H, Winckler W, Guttman M, Lander ES, Getz G, Mesirov JP (2011) Integrative genomics viewer. Nat Biotechnol 291:24-26

Rowińska E, Lukaszkiewicz J, Gradowska W, Lyson-Wojciechowska G, Proszynska K, Konopielko Z, Kulczycka H, Lorenc R, Pronicka E (1998) Abnormalities of calcium-phosphate metabolism in children with a past history of idiopathic infantile hypercalcemia. Ped Pol 73:23-30

Samuel HS (1964) Infantile Hypercalcaemia, nutritional rickets, and infantile scurvy in great Britain. A British Paediatric Association Report. Br Med J 15399:1659-1661

Schlingmann KP, Kaufmann M, Weber S, Irwin A, Goos C, John U, Misselwitz J, Klaus G, Kuwertz-Broking E, Fehrenbach H, Wingen AM, Guran T, Hoenderop JG, Bindels RJ, Prosser DE, Jones G, Konrad M (2011) Mutations in CYP24A1 and idiopathic infantile hypercalcemia. N Engl J Med 3655:410-421

Schlingmann KP, Ruminska J, Kaufmann M, Dursun I, Patti M, Kranz B, Pronicka E, Ciara E, Akcay T, Bulus D, Cornelissen EA, Gawlik A, Sikora P, Patzer L, Galiano M, Boyadzhiev V, Dumic M, Vivante A, Kleta R, Dekel B, Levtchenko E, Bindels RJ, Rust S, Forster IC, Hernando N, Jones G, Wagner CA, Konrad M (2016) Autosomalrecessive mutations in SLC34A1 encoding sodium-phosphate cotransporter 2A cause idiopathic infantile hypercalcemia. J Am Soc Nephrol 272:604-614

Wang K, Li M, Hakonarson H (2010) ANNOVAR: functional annotation of genetic variants from high-throughput sequencing data. Nucleic Acids Res 3816:e164 